\title{
Anti-Sway Control of the Overhead Crane System using HOSM Observer
}

\author{
Dongwoo Kwon*, Myunghwan Eom* and Dongkyoung Chwa ${ }^{\dagger}$
}

\begin{abstract}
This paper proposes a sum of squares (SOS) method for anti-swing control of overhead crane system using HOSM (High-Order Sliding-Mode) observer. By representing the dynamic equations of overhead crane as the polynomial dynamic equations via Taylor series expansion, the control input is obtained from the converted polynomial dynamic equations by numerical tool SOSTOOL. Since the actual crane systems include disturbance such as wind and friction, we propose a method to compensate for the disturbance by estimating the disturbance using HOSM observer. Numerical simulations show the effectiveness and the applicability of the proposed method.
\end{abstract}

Keywords: Sum of squares, Anti-swing crane control, Polynomial dynamic equations, Disturbance, HOSM Observer

\section{Introduction}

Overhead crane system that can move a heavy object has been much used in many construction sites and ports. One of the most important thing in crane system is to transfer payload as quickly and safely as possible within a given time. Sway of the heavy payload occurring when moving the trolley should be suppressed to achieve the efficient operation of the overhead crane system in that large sway of the payload causes the accident in industrial sites. Sway of the payload is directly affected by the length of the rope and acceleration of trolley. Thus, various researches to minimize sway of payload are in progress.

Crane control system has been studied using linear control, nonlinear control, and fuzzy control. The linear controller is designed by linearizing the nonlinear dynamic equations of the crane [1]. Although the linear controller is a very simple control method, it should be based on the linear model which is subject to the linearization error due to the omission of the nonlinear terms. In the case of nonlinear controller, a nonlinear control method based on feedback linearization for swing control ensures the stability which can be proved by Lyapunov stability theorem $[2,3]$. On the other hand, a fuzzy controller is to control sway of the crane by applying the expert knowledge. [4-7] proposed an algorithm using the Fuzzy LMI (Linear Matrix Inequality) technique in order to solve the stability problem of the system. The LMI-based algorithm requires a constant system matrix in order to calculate the control input. The performance of the controller is not guaranteed when such algorithm is applied to the actual model.

$\dagger$ Corresponding Author: Dept. of Electrical and Computer Engineering, Ajou University, Korea. (dkchwa@ajou.ac.kr)

* Dept. of Electrical and Computer Engineering, Ajou University, Korea. (\{opq0001, mgy777\}@ajou.ac.kr)

Received: December 14, 2015; Accepted: April 1, 2016
The actual crane systems include disturbance such as wind and friction. In the actual system, observation of the system states including the disturbance is one of the most important issues. HOSM observer designs for linear system with unknown input have been studied in various ways [8-11]. The corresponding conditions for linear timeinvariant systems with disturbances were obtained in [10, 13]. HOSM observer provides the global observation of the state and the output. In addition, observer provides global finite-time exact observation of the state and identification of smooth disturbances of strongly observable systems. High-order sliding-mode differentiators were suggested for exact observer design [14-17]. It provides the finite time convergence of the observation error in the existence of the disturbance.

In this paper, we show that nonlinear terms in the dynamic equations of the crane can be converted into the form of a polynomial matrix system using the Taylor series expansion without having to linearize the nonlinear terms. Then, we obtain the control input using the Sum of Squares (SOS) method [11]. The control input is obtained from the converted polynomial dynamic equations by using the numerical tool SOSTOOL [18]. Furthermore, the disturbance presented in the actual system and state vector are estimated by using the HOSM observer. Disturbances presented in each of the trolley traveling motion and sway motion can be compensated altogether by estimating only one of the disturbances in both systems using HOSM observer. In this way, we can improve the performance of the crane control system and then verify its performance through the stability analysis and the numerical simulations.

\section{Modeling of Overhead Crane}

Fig. 1 represents the 2-D model of the overhead crane. 


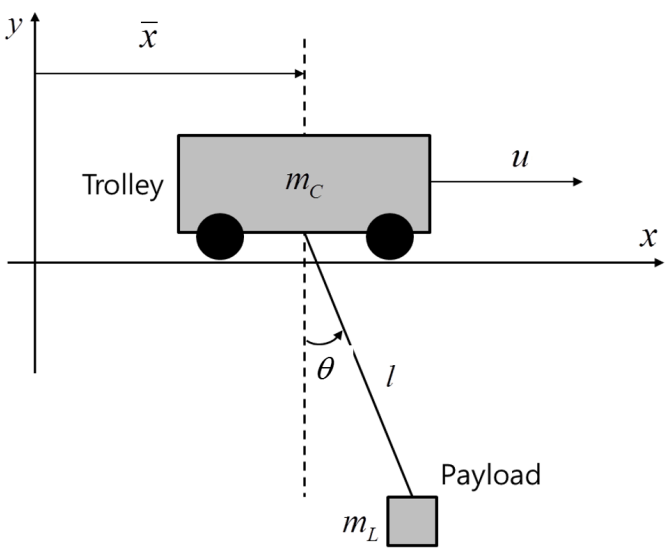

Fig. 1. 2-D model of an overhead crane $\left(m_{L}\right.$ : payload mass, $m_{C}$ : trolley mass, $l$ : rope length, $u$ : control input, $\bar{x}:$ trolley position, $\theta:$ sway angle)

By assuming that the load has a point mass, and the rope has no mass and elasticity, the dynamic equation of the crane can be expressed as [1]

$$
\begin{gathered}
\left(m_{L}+m_{C}\right) \ddot{\bar{x}}+m_{L} l \ddot{\theta} \cos \theta-m_{L} \dot{\theta}^{2} l \sin \theta=u \\
m_{L} l \cos \theta \ddot{\bar{x}}+m_{L} l^{2} \ddot{\theta}+m_{L} g l \sin \theta=0
\end{gathered}
$$

where $g$ is gravity constant. Considering the disturbance such as friction, the crane system in (1) can be rewritten as

$$
\begin{aligned}
& \left(m_{L}+m_{C}\right) \ddot{\bar{x}}+m_{L} l \ddot{\theta} \cos \theta-m_{L} \dot{\theta}^{2} l \sin \theta=u \\
& m_{L} l \cos \theta \ddot{\bar{x}}+m_{L} l^{2} \ddot{\theta}+m_{L} g l \sin \theta-\mu \dot{\theta}=0
\end{aligned}
$$

where $\mu$ is the viscous damping coefficient associated with the trolley motion. Eq. (2) can be arranged as

$$
\begin{aligned}
& \ddot{\bar{x}}=F_{x}+G_{x} u+\Omega_{x} \\
& \ddot{\theta}=F_{\theta}+G_{\theta} u+\Omega_{\theta}
\end{aligned}
$$

where

$F_{x}=\frac{W}{R}, G_{x}=\frac{P}{R}, F_{\theta}=\frac{V}{S}, G_{\theta}=\frac{Q}{S}$,

$\Omega_{x}=\frac{-\cos \theta}{R l}(\mu \dot{\theta}), \Omega_{\theta}=\frac{\left(m_{L}+m_{C}\right) \mu \dot{\theta}}{m_{L} l S}$,

$W=m_{L} l \dot{\theta}^{2} \sin \theta+m_{L} g \cos \theta \sin \theta$,

$V=m_{L} l \dot{\theta}^{2} \sin \theta \cos \theta+\left(m_{L}+m_{C}\right) g \sin \theta, \quad R=\left(m_{L}+m_{C}\right)$

$-m_{L} \cos ^{2} \theta, P=1, Q=\cos \theta$,

$S=l\left(\left(-m_{L}-m_{C}\right)+m_{L} \cos ^{2} \theta\right)$,

$\Omega_{x}$ and $\Omega_{\theta}$ are the lumped uncertainties of the actual crane system.

Unlike the linear systems, state variables should exist in the system matrix since dynamic Eqs. (1) and (2) contain the non-linear terms. Therefore, a lot of difference can exist between the actual model and the linearized model, which
Table 1. Taylor series expansion of nonlinear terms

\begin{tabular}{c|c}
\hline Nonlinear term & Taylor expansion \\
\hline $\sin \theta$ & $\theta-\frac{1}{6} \theta^{3}+\frac{1}{120} \theta^{5}$ \\
\hline $\cos \theta$ & $1-\frac{1}{2} \theta^{2}+\frac{1}{24} \theta^{4}-\frac{1}{720} \theta^{6}$ \\
\hline $\sin \theta \cos \theta$ & $\theta-\frac{2}{3} \theta^{3}+\frac{2}{15} \theta^{5}$ \\
\hline $\cos ^{2} \theta$ & $1-\theta^{2}+\frac{1}{3} \theta^{4}-\frac{2}{45} \theta^{6}$ \\
\hline
\end{tabular}

can be reduced by expressing nonlinear terms as polynomials by using Taylor series expansion as in Table 1 . Hence, polynomial equations can be obtained by using Taylor series expansion as

$$
\begin{aligned}
& \ddot{\bar{x}}=P_{1}+P_{2} u \\
& \ddot{\theta}=P_{3}+P_{4} u
\end{aligned}
$$

Where

$$
\begin{aligned}
& P_{1}=\frac{m_{L} g\left(\theta-\frac{2}{3} \theta^{3}+\frac{2}{15} \theta^{5}\right)+m_{L} l \dot{\theta}^{2}\left(\theta-\frac{1}{6} \theta^{3}+\frac{1}{120} \theta^{5}\right)}{m_{L}+m_{C}-m_{L}\left(1-\theta^{2}+\frac{1}{3} \theta^{4}-\frac{2}{45} \theta^{6}\right)} \\
& P_{2}=\frac{1}{m_{L}+m_{C}-m_{L}\left(1-\theta^{2}+\frac{1}{3} \theta^{4}-\frac{2}{45} \theta^{6}\right)} \\
& P_{3}=\frac{m_{L} l \dot{\theta}^{2}\left(\theta-\frac{2}{3} \theta^{3}+\frac{2}{15} \theta^{5}\right)+\left(m_{L}+m_{C}\right) g\left(\theta-\frac{1}{6} \theta^{3}+\frac{1}{120} \theta^{5}\right)}{l\left(-m_{L}-m_{C}+m_{L}\left(1-\theta^{2}+\frac{1}{3} \theta^{4}-\frac{2}{45} \theta^{6}\right)\right)} \\
& P_{4}=\frac{1-\frac{1}{2} \theta^{2}+\frac{1}{24} \theta^{4}+\frac{1}{720} \theta^{6}}{l\left(-m_{L}-m_{C}+m_{L}\left(1-\theta^{2}+\frac{1}{3} \theta^{4}-\frac{2}{45} \theta^{6}\right)\right.} .
\end{aligned}
$$

Then, the state space equations can be represented from (4) as follows:

$$
\left[\begin{array}{c}
\dot{\bar{x}} \\
\ddot{\bar{x}} \\
\dot{\theta} \\
\ddot{\theta}
\end{array}\right]=\left[\begin{array}{cccc}
0 & 1 & 0 & 0 \\
0 & 0 & \frac{P_{1}}{\theta} & 0 \\
0 & 0 & 0 & 1 \\
0 & 0 & \frac{P_{3}}{\theta} & 0
\end{array}\right]\left[\begin{array}{c}
\bar{x} \\
\dot{\bar{x}} \\
\theta \\
\dot{\theta}
\end{array}\right]+\left[\begin{array}{c}
0 \\
P_{2} \\
0 \\
P_{4}
\end{array}\right] u .
$$

The objective is to make the position error $e=\bar{x}-r$ for a constant reference position of the trolley $r$ converge to zero. Then, by introducing $x:=[e, \dot{e}, \theta, \dot{\theta}]^{T} \in \mathfrak{R}^{n}$ where $n=4$, the position error dynamics is represented as

$$
\dot{x}=A(x) x+B(x) u
$$


where $A(x)$ and $B(x)$ are the corresponding matrix and vector in (5).

The design procedure for the proposed SOS-based disturbance compensation control using HOSM observer consists of two steps; i.e., the SOS-based controller design and the HOSM observer design, which will be described in the following sections.

\section{SOS-based Controller Design}

In this section, we design the controller for the crane system presented in the previous section using SOSTOOL. A multivariate polynomial $p(x)$ is a sum of squares (SOS), if there exist polynomials $p_{1}(x), \ldots ., p_{m}(x)$ such that $p(x)=\sum_{i=1}^{m} p_{i}^{2}(x)$. It is clear that $p(x)$ being an SOS naturally implies $p(x) \geq 0$ for all $x(t) \in \mathfrak{R}^{n}$ [18]. Here, $p(x)$ is an SOS if there exists a positive semidefinite matrix $P(x)$ such that

$$
p(x)=\hat{x}(x)^{T} P(x) \hat{x}(x)
$$

where $\hat{x}(x) \in \mathrm{R}^{N \times N}$ is a column vector whose entries are all monomials in $x$. This immediately gives Lemma 1 .

Lemma 1 [19]: For a symmetric polynomial matrix $P(x)$ which is nonsingular for all $x$, we have

$$
\frac{\partial P}{\partial x_{i}}(x)=-P(x) \frac{\partial P^{-1}}{\partial x_{i}}(x) P(x) \text {. }
$$

Proof: Since $P(x)$ is nonsingular, we have $P(x) P^{-1}(x)=I$. Differentiating both sides with respect to $x_{i}$ yields

$$
\frac{\partial P}{\partial x_{i}}(x) P^{-1}(x)+P(x) \frac{\partial P^{-1}}{\partial x_{i}}(x)=0 .
$$

Then, the stability of the overall control system can be described as the following theorem.

Theorem 1: For the system in Eq. (6), suppose that there exist an $N \times N$ symmetric polynomial matrix $X^{-1}(\tilde{x})$, a $1 \times N$ polynomial vector $M(\tilde{x})$, an $n \times N$ polynomial matrix $\varepsilon_{1} \geq 0$, and an $\operatorname{SOS} \varepsilon_{2}$, such that the following two expressions hold.

$$
\begin{aligned}
& v^{T}\left(X(\tilde{x})-\varepsilon_{1}(x) I\right) v \text { is SOS } \\
& -v^{T}\left(\begin{array}{c}
T(x) A(x) X(\tilde{x})+X(\tilde{x}) A^{T}(x) T^{T}(x) \\
+T(x) B(x) M(\tilde{x})+M^{T}(\tilde{x}) A^{T}(x) T^{T}(x) \\
-\sum_{k \in J} \frac{\partial X(\tilde{x})}{\partial x_{k}} A^{k}(x)(\tilde{x})+\varepsilon_{2}(x) I
\end{array}\right) v \text { is SOS }
\end{aligned}
$$

where $T^{i j}(x)=\frac{\partial \hat{x}_{i}(x)}{\partial x_{j}}, \quad J=\left\{j_{1}, j_{2}, \ldots, j_{m}\right\}$ denote the row indices of $B(x)$ whose corresponding row is equal to zero, and $A^{k}(x)$ is the $k$ th row of $A(x)$ [19]. Then, the state feedback stabilization problem is solvable using a feedback control input $K_{S O S}(x) \hat{x}(x)$ where

$$
K_{S O S}(x)=M(\tilde{x}) X^{-1}(\tilde{x}) .
$$

Proof: Assume that there exist solutions $X(\tilde{x})$ and $M(\tilde{x})$ for Eqs. (10) and (11). Define the Lyapunov function $V(x)$ as

$$
V(x)=\hat{x}(x)^{T} X^{-1}(\tilde{x}) \hat{x}(x)
$$

Then, the time derivative of $V(x)$ along the closed-loop system trajectories is given by

$$
\begin{aligned}
\dot{V}(x)= & \dot{\hat{x}}^{T}(x) X^{-1}(\tilde{x}) \hat{x}(x)+\hat{x}^{T}(x) X^{-1}(\tilde{x}) \dot{\hat{x}}^{T}(x) \\
& +\hat{x}^{T}(x) \sum_{k \in J} \frac{\partial X^{-1}(\hat{x})}{\partial x_{k}} A^{k}(x) \hat{x}(x) .
\end{aligned}
$$

Substituting Eq. (6) into Eq. (14) can be expressed as

$$
\dot{V}(x)=\hat{x}^{T}(x)\left(\begin{array}{c}
K^{T}(x) B^{T}(x) X^{-1}(\tilde{x})+A^{T}(x) X^{-1}(\tilde{x}) \\
+X^{-1}(\tilde{x}) A(x)+X^{-1}(\tilde{x}) B(x) K_{S O S}(x)
\end{array}\right) \hat{x}(x) .
$$

Multiplying the above equation from the left and right by $X(\tilde{x})$, and using the result in Lemma 1, we can conclude that derivative of Lyapunov function is negative definite for all $x$ considering that $\varepsilon_{2}(x) I$ is included in the SOS condition in (11). This proves the stability of the closed-loop system.

\section{Design of HOSM Observer}

In this section, we discuss a HOSM observer for estimating the disturbance presented in the actual system. The HOSM observer is designed for linear system with bounded unknown inputs. Thus, linear system with disturbance $\zeta$ of the crane system in the vicinity of the desired equilibrium points can be expressed by using $\left(\dot{\theta}^{2} \approx 0, \cos \theta \approx 1, \sin \theta \approx \theta, \sin ^{2} \theta \approx 0\right)$ as

$$
\begin{aligned}
& \dot{x}=A x+B u+D \zeta \\
& y=C x
\end{aligned}
$$

where 
$A=\left[\begin{array}{cccc}0 & 1 & 0 & 0 \\ 0 & 0 & \frac{m_{L}}{m_{C}} g & 0 \\ 0 & 0 & 0 & 1 \\ 0 & 0 & \frac{-\left(m_{L}+m_{C}\right) g}{m_{C} l} & 0\end{array}\right], B=\left[\begin{array}{llll}0 & \frac{1}{m_{C}} & 0 & \frac{-1}{m_{C} l}\end{array}\right]^{T}$,

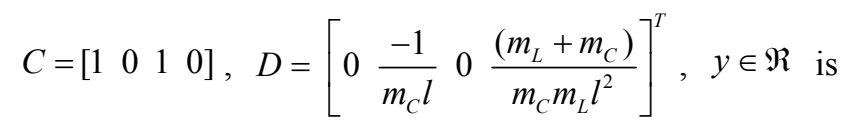

output, $\zeta:=\mu \dot{\theta} \in \mathfrak{R}$ is disturbance, and $u \in \mathfrak{R}$ is the control input obtained by the SOS approach as in the previous section. The matrix $C$ is related with the trolley position and sway angle and $D$ is related with the disturbance. In order to estimate the disturbance in the actual crane system, it is necessary to design an observer. Hence, we propose an algorithm which can estimate disturbance for linear system. Consider the crane system with disturbance, dynamic Eq. (1) is rewritten as

$\ddot{\bar{x}}=\frac{m_{L} g \cos \theta \sin \theta+m_{L} l \dot{\theta}^{2} \sin \theta-\frac{\cos \theta}{l} \mu \dot{\theta}+u}{\left(m_{L}+m_{c}\right)-m_{L} \cos ^{2} \theta}$

$\ddot{\theta}=\frac{m_{L} l \dot{\theta}^{2} \cos \theta \sin \theta+u \cos \theta+\left(m_{L}+m_{c}\right) g \sin \theta-\frac{\left(m_{L}+m_{C}\right)}{m_{L} l} \mu \dot{\theta}}{l\left(-m_{L}-m_{c}+m_{L} \cos ^{2} \theta\right)}$.

The structure of the proposed disturbance compensation method is shown in Fig. 2. Here, the disturbance estimate $\hat{\zeta}$ will be obtained in the following subsections.

\subsection{State estimation}

The crane system containing the disturbance $\zeta$ in (16) has the relative degree $n$ with respect to the disturbance $\zeta$. To estimate the states, the observer is designed in the following form [14]:

$$
\begin{gathered}
\dot{z}=A z+B u+L(y-C z), \\
\hat{x}=x+K v,
\end{gathered}
$$

$$
\dot{v}=W(y-C z, v),
$$

where $\hat{x}$ is the estimate of $x$. The column matrix $L$ is a correction factor designed so that the eigenvalues of a matrix $A-L C$ have negative real parts. The gain matrix $K=P^{-1}$ and $P$ can be written as

$$
P=\left[\begin{array}{c}
C \\
C(A-L C) \\
C(A-L C)^{2} \\
\vdots \\
C(A-L C)^{n-1}
\end{array}\right]
$$

and vector $v$ and the nonlinear discontinuous function $W=\left\{w_{1}, w_{2}, \ldots, w_{n-1}\right\}$ are selected differently.

Eq. (20) is the high-order sliding mode differentiator. The derivatives of the measured outputs can be estimated in finite time by the high-order sliding mode differentiator [16]. $(n-1)$ th-order differentiator can be written in the form

$$
\begin{aligned}
& \dot{v}_{1}=w_{1}=-\alpha_{n} M^{1 / n} \mid v_{1}-y \\
& +\left.C z\right|^{(n-1) / n} \operatorname{sign}\left(v_{1}-y z+C z\right)+v_{2} \\
& \dot{v}_{2}=w_{2}=-\alpha_{n-1} M^{1 /(n-1)} \mid v_{2} \\
& \quad+\left.w_{1}\right|^{(n-2) /(n-1)} \operatorname{sign}\left(v_{2}-w_{1}\right)+v_{3}, \\
& \vdots \quad \\
& \dot{v}_{n-1}=w_{n-1}=-\alpha_{2} M^{1 / 2} \mid v_{n-1} \\
& \quad+\left.w_{n-2}\right|^{1 / 2} \operatorname{sign}\left(v_{n-1}-w_{n-2}\right)+v_{n,} \\
& \dot{v}_{n}=-\alpha_{1} \operatorname{Mign}\left(v_{n}-w_{n-1}\right),
\end{aligned}
$$

where $v_{i}, z_{i}$, and $w_{i}$ are the components of the vector $v, \quad z \in \mathfrak{R}^{n}$, and $w \in \mathfrak{R}^{n-1}$, respectively; $M$ is a sufficiently large parameter, the constant $\alpha_{i}$ is chosen recursively sufficiently large as $\alpha_{1}=1.1, \alpha_{2}=1.5$, $\alpha_{3}=2, \alpha_{4}=3, \alpha_{5}=5$, and $\alpha_{6}=8$ [16].

In this way, the asymptotic estimate value $\hat{e}$ can be obtained by designing the Luenberger observer method and

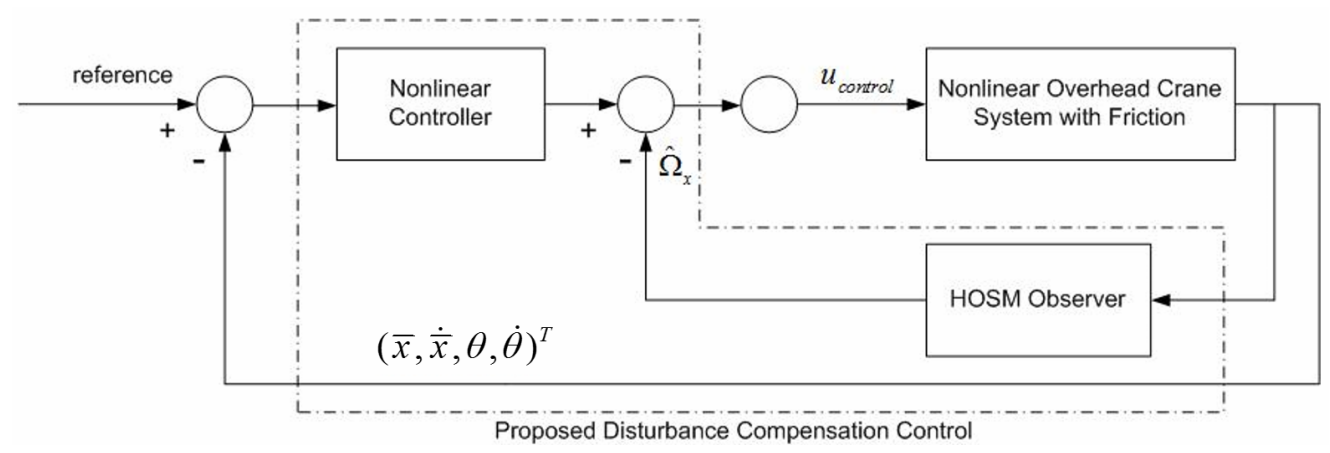

Fig. 2. Structure of the proposed disturbance compensation control system 
high-order sliding mode differentiator that ensures the finite time convergence.

\subsection{Disturbance estimation}

We can obtain internal variables $w_{i}$ in (22) using the values of $y, z$ and $v_{i}, i=1, \ldots, n$. Therefore, the observer equation

$$
\hat{x}=z+P w
$$

is used instead of equation (19). The estimation of the disturbance $\hat{\zeta}$ is obtained as

$$
\begin{aligned}
& \hat{\zeta}=\frac{1}{d}\left(v_{n+1}-\left(a_{1} v_{1}+a_{2} v_{2}+\cdots+a_{n} v_{n}\right)\right), \\
& s^{n}-a_{n} s^{n-1}-\cdots-a_{1}=(-1)^{n} \operatorname{det}(A-L C-s I)
\end{aligned}
$$

where $d=C A^{n-1} D$ and $I$ is an identity matrix. Thus, we can create a disturbance in the crane system as

$$
\hat{\Omega}_{x}=G_{x} \hat{\zeta} .
$$

Eq. (3) has two uncertainties $\Omega_{x}$ and $\Omega_{\theta}$, which are coupled with each other due to the underactuation characteristics of the crane as

$$
\Omega_{x}=-\Psi \Omega_{\theta}
$$

where $\Psi=-m_{L} l^{2} / m_{L} l \cos \theta$. Therefore, disturbance of the system presented in each of the trolley traveling motion and sway motion can be compensated altogether by estimating only one of the disturbances in both systems using HOSM observer. By defining acceleration uncertainties as $\Omega_{x}$ and $\Omega_{\theta}$, the overhead crane system model in (3) can be rewritten as

$$
\begin{aligned}
\ddot{x}-\Omega_{x} & =F_{x}+G_{x} u \\
\ddot{\theta}-\Omega_{\theta} & =F_{\theta}+G_{\theta} u
\end{aligned} .
$$

Thus, by substituting $\ddot{x}-\Omega_{x}$ and $\ddot{\theta}-\Omega_{\theta}$ into Eq. (1), we can have

$$
\begin{gathered}
m_{L} l \cos \theta\left(\ddot{x}-\Omega_{x}\right)+m_{L} l^{2}\left(\ddot{\theta}-\Omega_{\theta}\right)+m_{L} g l \sin \theta=0 \\
-m_{L} l \cos \theta \Omega_{x}=m_{L} l^{2} \Omega_{\theta}
\end{gathered}
$$

which leads to Eq. (26). Thus, by combining compensation input in (25) and the SOS-based control input $u_{\text {control }}=K_{S O S}(x) \hat{x}(x)+\hat{\Omega}_{x} \quad$ for the crane system with disturbance, the disturbance can be compensated, which is verified through simulation results in the next section.

\section{Numerical Simulations}

In this section, we present simulation results to verify the performance of the proposed control and disturbance compensation methods. The parameters of the crane are given as $m_{C}=1000 \mathrm{~kg}, m_{L}=1500 \mathrm{~kg}, l=8 \mathrm{~m}$, and $g=9.8 \mathrm{~m} / \mathrm{s}^{2}$. The target position was set to $10 \mathrm{~m}$. The proposed anti-sway control method based on the estimation of the disturbance presented in the crane system consists of the design of HOSM observer and the disturbance compensation controller. For the linear crane system (16), the correction factor $L=[-64.5,178.5,91.5,85.7]^{T}$ provides the eigenvalues $-5,-8.5,-5,-8.5$ of the matrix $A-L C$. The matrices $X^{-1}(\tilde{x})$ and $M(\tilde{x})$ obtained by using Theorem 1 can be written as follows:

$$
\begin{gathered}
X^{-1}(\tilde{x})=P\left(=P^{T}\right)=\left(\begin{array}{llll}
P_{11} & P_{12} & P_{13} & P_{14} \\
P_{12} & P_{22} & P_{23} & P_{24} \\
P_{13} & P_{23} & P_{33} & P_{34} \\
P_{14} & P_{24} & P_{34} & P_{44}
\end{array}\right) \\
M(\tilde{x})=\left[M_{1}, M_{2}, M_{3}, M_{4}\right]
\end{gathered}
$$

where

$$
\begin{aligned}
& P_{11}= 0.023073 x_{3}^{2}-0.0015963 x_{3} x_{4}+2.5695 \\
& P_{12}=-0.00445 x_{3}^{2}+0.0021635 x_{3} x_{4}-0.30356 \\
& P_{13}=-0.00023872 x_{3}^{2}-0.00021165 \\
& P_{14}=0.00031793 x_{3}^{2}-0.00096435 \\
& P_{22}= 0.015922 x_{3}^{2}-0.005689 x_{3} x_{4}+0.054714 \\
& P_{23}=-0.00062463 x_{3}^{2}+0.0013221 \\
& P_{24}=-0.0024015 x_{3}^{2}-0.0012296 \\
& P_{33}=0.00033881 x_{3}^{2}-0.00041309 x_{3} x_{4}+0.00028211 \\
& P_{34}=0.00054298 x_{3}^{2}+0.00021033 x_{3} x_{4}-0.000065039 \\
& P_{44}=0.00039005 x_{3}^{2}+0.0000035242 x_{3} x_{4} \\
& \quad+0.000054894 x_{4}^{2}+0.00054862 \\
& M_{1}=-0.591 x_{3}^{2}-0.0897 x_{3} x_{4}+1.68 x_{4}^{2}-1.54 \\
& M_{2}=-6.48 x_{3}^{2}+1.63 x_{3} x_{4}-20.8 x_{4}^{2}-25.3 \\
& M_{3}=-0.942 x_{3}^{2}+0.645 x_{3} x_{4}-2.34 x_{4}^{2}-2.71 \\
& M_{4}=0.645 x_{3}^{2}-2.34 x_{3} x_{4}+0.841 x_{4}^{2}+2.41 .
\end{aligned}
$$

Also, the HOSM differentiator can be written as

$$
\begin{aligned}
& \dot{v}_{1}=w_{1}=-8\left|v_{1}-y(t)+C_{Z}\right|^{5 / 6} \operatorname{sign}\left(v_{1}-y(t)+C z\right)+v_{2}, \\
& \dot{v}_{2}=w_{2}=-5\left|v_{2}-w_{1}\right|^{4 / 5} \operatorname{sign}\left(v_{2}-w_{1}\right)+v_{3}, \\
& \dot{v}_{3}=w_{3}=-3\left|v_{2}-w_{1}\right|^{3 / 4} \operatorname{sign}\left(v_{3}-w_{2}\right)+v_{4}, \\
& \dot{v}_{4}=w_{4}=-2\left|v_{3}-w_{2}\right|^{2 / 3} \operatorname{sign}\left(v_{4}-w_{3}\right)+v_{5}, \\
& \dot{v}_{5}=w_{5}=-1.5\left|v_{5}-w_{4}\right|^{1 / 2} \operatorname{sign}\left(v_{5}-w_{4}\right)+v_{6}, \\
& \dot{v}_{6}=-1.1 \operatorname{sign}\left(v_{6}-w_{5}\right) .
\end{aligned}
$$




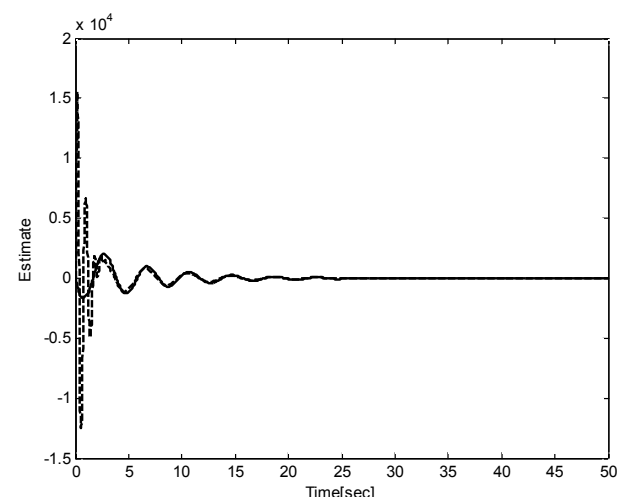

(a) Disturbance estimation (solid line: disturbance $\zeta$, dashed line: disturbance estimate $\hat{\zeta}$ )

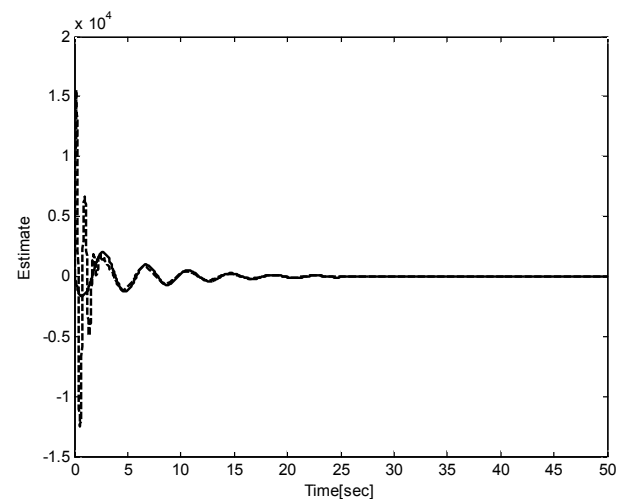

(c) Trolley position (solid line: compensated, dashed line: uncompensated)

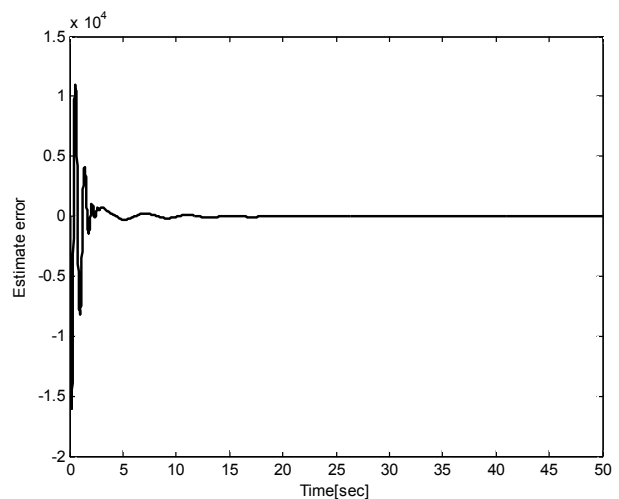

(b) Disturbance estimation error

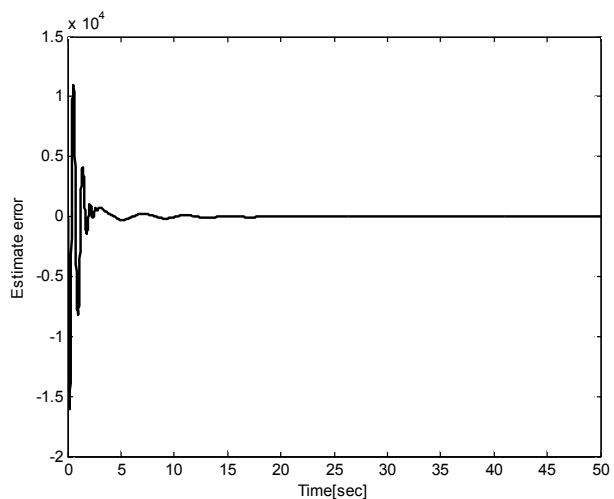

(d) Sway angle of payload (solid line: compensated, dashed line: uncompensated)

Fig. 3. Performance of the proposed method

The disturbance estimate $\hat{\Omega}_{x}$ is obtained in Eq. (24). In Fig. 3, we can see the observer performance and finite-time convergence of $\hat{\Omega}_{x}$ to $\Omega_{x}$. The estimation error between $\hat{\Omega}_{x}$ and $\Omega_{x}$ in Fig. 3 shows that SOS-based disturbance compensation control can be obtained by obtaining $\hat{\Omega}_{x}$ using the HOSM observer. When there is no compensation of disturbance, the control performance is much degraded. On the other hand, the influence of the disturbance presented in the system is removed using the proposed method. These results verify the advantages of the proposed algorithm.

\section{Conclusion}

In this paper, we proposed SOS-based control method and HOSM observer design for the anti-sway control of the overhead crane system with disturbance. First, we considered separately the disturbance in the trolley traveling motion and sway motion of the overhead crane system. By estimating the disturbance in the sway motion via HOSM observer, we have shown that it is possible to compensate both the disturbances in the entire system.
Then, we obtained the SOS-based anti-sway controller of the overhead crane system using the disturbance estimate. HOSM observer has advantages in that global finite-time exact observation of the state and finite-time exact identification of disturbance can be achieved by using HOSM differentiator. We have shown that the proposed methods can maintain the satisfactory performance of the overhead crane system with disturbance.

\section{Acknowledgements}

This work was partly supported by the ICT R\&D program of MSIP/IITP [14-824-09-013, Resilient CyberPhysical Systems Research)] and also supported by the National Research Foundation of Korea under a grant supported by the Korea government (MSIP) (2014 R1A2A1A11053153).

\section{References}

[1] A. Piazzi and A. Visioli, "Optimal dynamic inversion 
based control of an overhead crane," IEE Proceeding of the Control Theory and Application, Vol. 149, No. 5, Sep. 2002, pp. 405-411.

[2] H. Park, D, Chwa, and K-.S. Hong, "A feedback linearization control of container cranes: varying rope length," International Journal of Control, Automation, and System, Vol. 5, No. 4, Aug. 2007, pp. 379-387.

[3] Q. H. Ngo, K.-S. Hong, "Sliding-Mode Antisway Control of an Offshore Container Crane," IEEE/ ASME Transactions on Mechatronics, vol. 17, no. 2, Apr. 2012, pp. 201-209.

[4] K. Tanaka, H. Ohtake, and O. Wang, "A Descriptor System Approach to Fuzzy Control System Design via Fuzzy Lyapunov Function," IEEE Transactions on Fuzzy System, Vol. 15, Jun. 2007, pp. 331-341.

[5] K. Tanaka, H. Yoshida, and H. Ohtake, O. Wang, "A Sum of Squares Approach to Stability Analysis of Polynomial Fuzzy System," American Control Conference, New York, Jul. 2007.

[6] Y. Zhao, H. Gao, "Fuzzy-Model-Based Control of an Overhead Crane With Input Delay and Actuator Saturation," IEEE Transactrions on Fuzzy System, vol. 20, no. 1, Feb. 2012, pp. 181-186.

[7] Y-J. Chen, W-J. Wang, and C-L. Chang, "Guaranteed cost control for an overhead crane with practical constraints: Fuzzy descriptor system approach," Engineering Applications of Artificial Intelligence, vol. 22, no. 4-5, Jun. 2009, pp. 639-645.

[8] V. Utkin, J. Guldner, and J. Shi, "Sliding Mode control in Electro-mechanical Systems," Taylor \& Francis: London, 1999, pp. 103-115.

[9] C. Edwards, S. Spurgeon, "Sliding Mode Control," Taylor \& Francis: London, 1998.

[10] T. Boukhabza, M. Djemai, J. Barbot, "Implicit Triangular Observer Form Dedicated to a Sliding Mode Observer for Systems with Unknown Input," Asian Journal of Control, vol. 5, no. 4, Dec. 2003, pp. 513527

[11] J. Davila, L. Fridman, A. Levant, "Second-order sliding-mode observer form mechanical systems," IEEE Transactions on Automatic Control, vol. 50, no. 11, 2005, pp. 1785-1789.

[12] R. C. Baker and B. Charlie, "Nonlinear unstable systems," International Journal of Control, Automation, and Systems, vol. 23, no. 4, May. 1989, pp. 123-145.

[13] L. Fridman, A. Levant, J. Davila, "High-order sliding-mode observation and identification for linear systems with unknown inputs," Proceedings of the $45^{\text {th }}$ Conference on Decision in Control, San Diego, CA, USA, Dec. 2006, pp. 5567-5572

[14] A. Levant, "High-order sliding modes: differentiation and output-feedback control," International Journal of Control, vol. 76, no. 9/10, 2003, pp. 924-941.

[15] F. F. Bejarano, L. Fridman, "High order sliding mode observer for linear systems with unbounded unknown inputs," International Journal of Control, vol. 83, no. 9, Sep. 2010, pp. 1920-1929.

[16] L. Fridman, A. Levant, and J. Davila "Observation of linear systems with unknown inputs via high-order sliding-modes," International Journal of Systems Science, vol. 38, no. 10, Oct. 2007, pp. 773-791.

[17] A. Levant, "High-order sliding modes: differentiation and output-feedback control," International Journal of Control, Vol.76, 2003, pp. 924-941.

[18] S. Prajna, A. Papachristodoulou, P. Seiler, and P. A. Parrilo, "SOSTOOLS: Sum of Squares Optimization Toolbox for MATLAB," Version 2.00, California Institute of Technology, Pasadena, CA, 2004.

[19] S. Prajna, A. Papachristodoulou, and F. Wu, "Nonlinear control synthesis by sum of squares optimization: A Lyapunov-based approach," Proceedings of Asian Control conference, Melbourne, Victoria, Australia, Jul. 2004.

[20] A. Piazzi and A. Visioli, "Optimal dynamic inversion based control of an overhead crane," IEE Proceedings of the Control Theory and Applications, vol 149, no. 5, Sep. 2002, pp. 405-411.

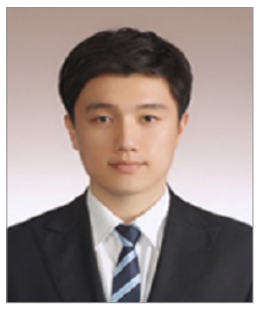

Dongwoo Kwon $\mathrm{He}$ received the B.S., M.S. degrees in electrical and computer engineering from Ajou University, Suwon, Korea, in 2014 and 2016, respectively. His research interests include nonlinear control and disturbance observer theories and their applications to cyber physical systems

(CPS) and cranes.

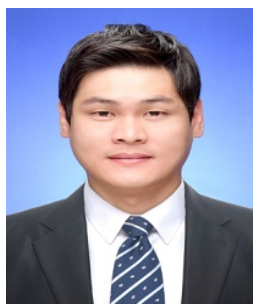

Myunghwan Eom $\mathrm{He}$ received the B.S., M.S., and Ph.D. degrees in electrical and computer engineering from Ajou University, Suwon, Korea, in 2009, 2011, and 2016, respectively. $\mathrm{He}$ is currently a Postdoctoral Researcher with Ajou University. His research interests include adaptive and robust nonlinear control theories and disturbance observer theories and their applications to robotics and underactuated mechanical systems, including pendubot, cranes, and autonomous unmanned vehicles. 


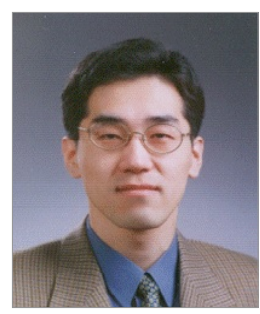

Dongkyoung Chwa He received the B.S. and M.S. degrees in control and instrumentation engineering and the $\mathrm{Ph} . \mathrm{D}$. degree in electrical and computer engineering from Seoul National University, Seoul, Korea, in 1995, 1997, and 2001, respectively. From 2001 to 2003, he was a Postdoctoral Researcher with Seoul National University, where he was also a BK21 Assistant Professor in 2004. Since 2005, he has been with the Department of Electrical and Computer Engineering, Ajou University, Suwon, Korea, where he is currently a Professor. He was a Visiting Scholar with the University of New South Wales at the Australian Defence Force Academy and the University of Melbourne, Melbourne, Vic., Australia, in 2003 and the University of Florida, Gainesville, in 2011. His research interests include nonlinear, robust, and adaptive control theories and their applications to robotics; underactuated systems, including wheeled mobile robots; underactuated ships; cranes; and guidance and control of flight systems. 